\title{
LA DEMANDA DE PROFESIONALES UNIVERSITARIOS EN EL CONTEXTO NACIONAL: UNA VISIÓN DESDE LA EMPRESA
}

Juan Enrique Froemel* 


\section{RESUMEN}

El artículo enfrenta el tema de la demanda de profesionales universitarios en Chile, vista desde la óptica primaria de la empresa. Se inicia con una discusión acerca de la posibilidad de dimensionar tal demanda, su pertinencia y legitimidad. Entrega, también, antecedentes acerca de la influencia de algunas tendencias internacionales en el empleo, y en las características de los profesionales. Continúa con un análisis acerca de los roles del pregrado y del posgrado en la formación de profesionales, revisa tipos y concepciones de tal formación. Comenta los rasgos más destacados de los profesionales chilenos a futuro y finaliza con una breve consideración respecto a la actitud de las universidades chilenas frente a la demanda de profesionales.

\section{ABSTRACT}

The article addresses the issue of the demand for professionals with university graduate degrees in Chile viewing the question primarily from the standpoint of companies. The paper begins with a discussion on the possibility of measuring demand, its appropriateness and legitimacy. It also provides background data on the influence that some international trends have on employment, and the distinctive features of professionals. It then goes on to analyze the roles played by undergraduate and graduate studies in the training of professionals, and it reviews the types and concepts that underlie such training. The article examines the most outstanding features of Chilean professionals for the future, and ends with a brief reflection on the attitude of Chilean universities vis-à-vis the demand for professionals. 


\section{LA DEMANDA DE PROFESIONALES UNIVERSITARIOS EN EL CONTEXTO NACIONAL: UNA VISIÓN DESDE LA EMPRESA}

LA REAL POSIBILIDAD DE DIMENSIONAR LA DEMANDA DE PROFESIONALES. LA EFICACIA, PERTINENCIA Y LEGITIMIDAD DE LAS ESTIMACIONES DE ELLA EN LA FORMACIÓN DE PROFESIONALES

Al igual que en cualquier otro lugar del mundo, la primera inquietud que surge al analizar la posibilidad real de dimensionar la demanda de profesionales universitarios en Chile desde la particular perspectiva de la empresa, corresponde a que la experiencia indica que, las más de las veces, el resultado de este tipo de pronósticos no guarda mayor relación con la demanda efectiva que se pueda producir más tarde. Es recomendable en este aspecto como en muchos otros del campo del estudio de ésta que, si bien es posible hacer estimaciones del mercado futuro, se debe ser en extremo cuidadoso a la hora de utilizar dicha información para efectos de planificación. Lo anterior no significa una renuncia de plano a la posibilidad de realizar análisis prospectivos de la demanda, pero se debe tener claro que tales pronósticos son limitados respecto de los productos de la Educación, como en la mayoría de los temas que se relacionan con el área social. Tal limitación obedece principalmente a dos factores: la gran cantidad de variables relacionadas con el campo educativo y las complejísimas relaciones entre ellas.

Lo señalado, conduce directamente al segundo tema de esta sección, cual es la cuestionable eficacia de tales proyecciones. Acerca de ella existen ejemplos evidentes, como las proyecciones que a mediados de los setenta efectuaran las universidades chilenas en el área de Administración de Negocios y Finanzas -en pregrado, Ingeniería Comercial-, a raíz de las nuevas orientaciones económicas 
introducidas en el país, en los primeros años del régimen militar. Tales proyecciones se vieron contradichas en la realidad por la crisis económica de 1978. Entonces, esas predicciones resultaron en un superávit manifiesto de profesionales en el rubro, con el consiguiente subempleo de los mismos.

Sin perjuicio de las limitaciones para determinar esta demanda en el nivel nacional, vale la pena explorar ciertos factores que en el ámbito internacional parecen mostrar un efecto sobre la pertinencia de ella. Estos factores son dos:

1) la evolución del mercado laboral, concretamente de la demanda, en los países desarrollados y

2) la incipiente, aunque en aumento, demanda de graduados en los segmentos más avanzados de los sistemas educativos por parte de las empresas, en especialidades no relacionadas con la tecnología ni con la administración de negocios, antiguamente reservadas a entidades de orientación más académica.

Algunos datos acerca de la evolución de la demanda de empleo en los países de la Organización de Desarrollo y Cooperación Económica $(\mathrm{OCDE})^{1}$ aparecen en el Informe del Estudio Internacional de Alfabetismo de Adultos (Adult Literacy Survey, IALS), que se realizó a fines de los noventa, titulado Literacy in the Information Age (OECD, 2000). El estudio señala que las empresas más competitivas hoy en día son aquellas que privilegian la adopción de prácticas flexibles de administración, las cuales incluyen mayor rotación que la habitual en los puestos de trabajo; un diseño de funciones que requieren que sus empleados reúnan múltiples destrezas de manera simultánea; gran énfasis en el trabajo en equipo y una coincidente reducción de los niveles jerárquicos, apoyada por una notoria delegación de responsabilidades en individuos y equipos de trabajo.

Simultáneamente, el Informe señala que la investigación en países como Australia y Estados Unidos muestra que tales empresas

1 Los países que integran la OCDE son: Alemania, Australia, Bélgica, Canadá, Corea, Dinamarca, España, Eslovaquia, Estados Unidos de Norteamérica, Finlandia, Francia, Grecia, Holanda, Hungría, Irlanda, Islandia, Italia, Japón, Luxemburgo, México, Noruega, Nueva Zelanda, Polonia, Portugal, Reino Unido, República Checa, Suecia, Suiza y Turquía. 
privilegian la contratación de empleados con mayores destrezas y con más altos niveles de educación. Agrega que, a los efectos de estos cambios organizacionales, se unen los efectos de la globalización y de la creciente presencia de la tecnología, que hacen imprescindible a las empresas, para poder competir en el plano internacional, adaptarse a las nuevas tecnologías y aumentar su productividad y eficiencia. Para ello deben contar con empleados poseedores de más altas destrezas. El aumento del nivel educacional de los países de la OCDE se ha convertido en ambas, en la causa y la consecuencia de estos cambios.

Un aspecto señalado por el Informe es el aumento de las tasas de desempleo para las personas con menores niveles educativos en los países de la OCDE. Asimismo otro elemento revela (figura 1) cómo el aumento de los puestos de trabajo profesionales en las empresas manufactureras y de servicios se debe tanto al aumento global de estas actividades, como a la creciente complejidad de ellas. De este modo, se incrementa la demanda por empleados con mayores destrezas y con más altos niveles de educación. Queda claro, también (figura 2) que el único tipo de empleo que presenta un aumento relativo en los países de la OCDE, entre 1980 y 1996, es el que se distingue por la aplicación de altos niveles de tecnología, relacionado con una mayor preparación.

Figura $\mathrm{N}^{\circ} 1$

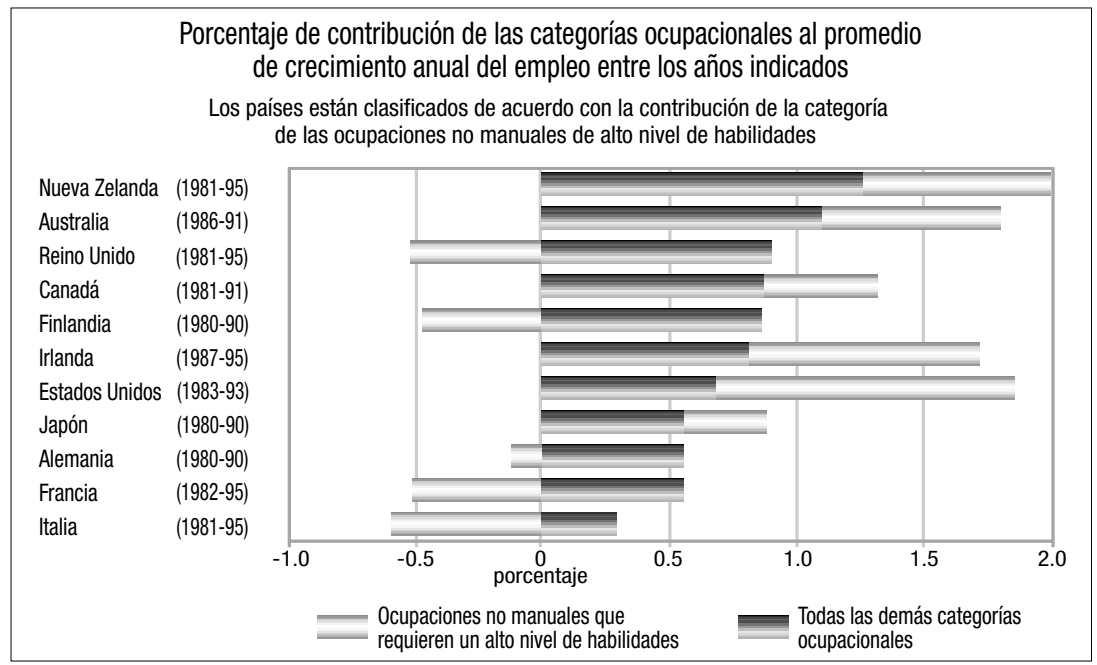

Fuente: OECD 1993a. 
Figura $\mathrm{N}^{\circ} 2$

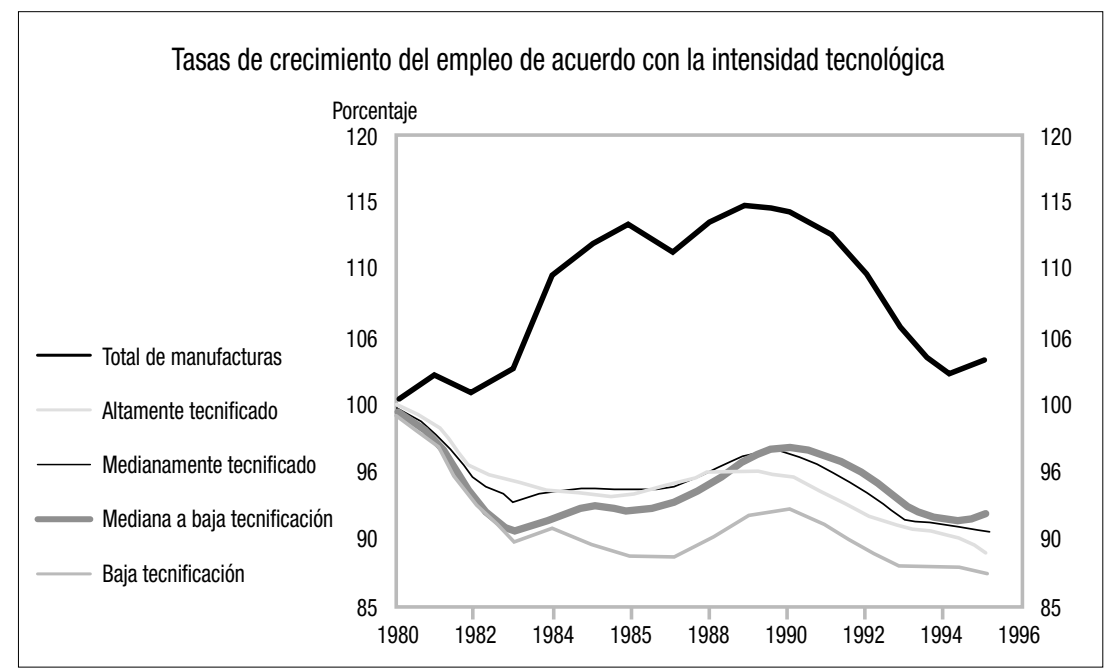

Fuente: OECD

El segundo factor considerado se refiere al aumento de la demanda de las empresas -incipiente aun- por profesionales de áreas no tradicionales, por llamarlas así. Hace algunos años la revista Time anticipó que las empresas de mayor tamaño en los países desarrollados iniciaron hace una década la contratación para cargos ejecutivos de graduados y posgraduados de profesiones y programas del área relacionada con las Humanidades y no de la vinculada con el mundo de los negocios ni de la empresa. Las nuevas temáticas más recurrentes entre estos han sido la Antropología, la Filosofía, la Historia y, en una segunda opción, aquellas como la Psicología y la Sociología. A fines de los noventa, Chile conoció un fenómeno parecido, de menor magnitud, aunque más ligado a áreas académicas propias de la Matemática y de las Ciencias Básicas, que incluyó, mayoritariamente, a egresados de programas de doctorados. Esta tendencia revela que ciertos rasgos que poseen los profesionales y posgraduados de las Humanidades y, eventualmente, de la Matemática y de las Ciencias Básicas, no son necesariamente patrimonio de quienes provienen del campo de los Negocios o de la Ingeniería. La publicación Time destacó la capacidad de análisis, basada en evidencia no cuantitativa y la apreciación de consecuencias de largo plazo, a partir de las alternativas de decisión posibles, características propias de las disciplinas humanísticas. En el caso chileno, las personas relacionadas con las Ciencias Básicas y la Matemática se distinguen por la capacidad de 
aplicación del método científico a problemas de corte táctico y estratégico en el marco de la empresa.

Ambas situaciones llaman a reflexionar en dos sentidos a la hora de analizar la demanda de profesionales por parte de la empresa en nuestro país. El primero de ellos considera que la actual formación de profesionales y posgraduados en el área de negocios contemple como metas deseables la incorporación de algunas competencias propias de los egresados de Humanidades, Matemática y Ciencias Básicas. El segundo, en cuanto a incentivar la demanda por parte de las empresas de los profesionales y posgraduados del área del conocimiento. Una manera práctica de acercarlos al mundo de los negocios sería incorporar en su formación algunos módulos relacionados con esta materia.

Cabe hacer ahora algunos alcances respecto de la legitimidad que pudiese tener la determinación de la demanda. A lo menos, existen dos ámbitos en los cuales es importante discutir tal legitimidad. El primero corresponde al campo ético en relación con la libertad de que deben disponer las personas para planificar sus vidas y, en particular, sus alternativas de formación. Corresponde preguntarse hasta dónde es válido introducir acciones de Ingeniería Social en un campo pleno de opciones para elegir un futuro educativo y laboral. Es válido preguntarse qué entidad está facultada para incentivar o desincentivar la elección de unas profesiones u oficios. ¿Es el Estado el facultado para ello, guiándose en las proyecciones económicas del respectivo país, o son las universidades las que deberían basarse en su misión e intereses académicos para determinar estas opciones? Independiente de los fundamentos de tales acciones, ies legítimo que las ejecute alguna entidad que no sean las personas involucradas en la elección?

Lo señalado lleva a concluir que para establecer una visión medianamente eficaz de la demanda de profesionales universitarios en Chile por parte de la empresa, más allá de concentrar la atención en variaciones nacionales, conviene observar con atención las tendencias mostradas por los países desarrollados en los últimos años.

\section{LA NECESARIA DISTINCIÓN ENTRE EL PREGRADO Y EL POSGRADO EN EL DESEMPEÑO LABORAL.}

La universidad chilena respondió por años a un perfil mayoritariamente profesionalizante, es decir, enfocó su rol docente a entregar 
profesionales al país, en un esquema heredero de la universidad napoleónica que, en el caso chileno, implicó un sesgo claro a favor del pregrado. Sin embargo, en el último medio siglo se aprecia un viraje importante, cuyo resultado es un énfasis creciente en la investigación $y$, consecuentemente, en el posgrado. Si bien existe un debate sostenido respecto de lo limitadas que son las dimensiones de este último, al igual que las de la actividad de investigación en Chile, es innegable que el país vivió un desarrollo creciente de ambos en los últimos veinte años.

No obstante, es necesario reconocer que las características propias del pregrado de las universidades chilenas de hace veinte o más años no son necesariamente las mismas de hoy. Esto implica que aunque ha mantenido un sello enfocado primariamente hacia las profesiones, incorporó como subsistema y, también, al interior de las carreras mismas, modificaciones importantes a la hora de analizar sus proyecciones laborales. Primero, el pregrado muestra hoy variantes, entre las que destacan, por su novedad y diversidad respecto de sus expresiones tradicionales, los programas que centran las etapas iniciales en la formación general, como el Bachillerato, de indudable raigambre anglosajona.

Por otra parte, en las carreras de pregrado de corte más tradicional también se advierten cambios que en su estructura las alejan de sus formas originales. Básicamente, estos se orientan a modificar las mallas curriculares con el fin de generar mayores espacios de flexibilidad y otorgar, al menos en sus etapas terminales, un mayor énfasis a la investigación.

Ambas situaciones han redundado en la formación de un profesional con un espectro mayor de competencias y una visión más madura de las razones que subyacen a su vocación por un área específica. De mantenerse esta tendencia, sería de esperar que este nuevo prototipo de profesional chileno respondiera cada vez de mejor manera a las exigencias de un mercado laboral que necesita ejecutivos adaptables a requerimientos cambiantes y con una disposición a complementar, e incluso reorientar, su formación universitaria inicial.

Sin perjuicio de reconocer la validez de este enunciado, es preciso tener presente que hay aun y seguirán existiendo diferencias marcadas 
entre el perfil de un profesional egresado del pregrado y quien ha complementado éste con el posgrado. Tales diferencias no son tan importantes cuando se compara la licenciatura con la maestría. Ello por cuanto la maestría en Chile corresponde a una especialización con un enfoque más profesional que académico. Resulta más evidente en la oferta de maestrías denominadas profesionales, representadas desde siempre en el ámbito de las ciencias empresariales por la maestría en administración de negocios (MBA).

La gran diferencia entre los profesionales con formación de pregrado y aquellos con posgrado, se produce más bien al momento del doctorado. En los países desarrollados ya no es novedad que en la alta gerencia de empresas privadas y públicas exista una demanda importante de graduados universitarios con posgrado y en particular de quienes poseen un doctorado. Más aún, no necesariamente las áreas de estudio coinciden siempre con el área o rubro de las empresas que los contratan. Un ejemplo interesante en este sentido proviene de las instituciones de la Defensa. En países como Estados Unidos existe la práctica, si bien no siempre la exigencia formal, que quienes ejerzan sus mandos superiores detenten un posgrado en temas no castrenses. Sin ir más lejos, en Chile se hace cada vez más habitual esta práctica. Así, en la actualidad, dos de tres de los más altos mandos de las Fuerzas Armadas finalizaron estudios de posgrado en instituciones universitarias civiles.

Hoy, la dirección de empresas e instituciones evoluciona en sus necesidades de personal hacia perfiles que se acercan más a una formación general, que implica gran flexibilidad y capacidad para integrar áreas del conocimiento muy diversas, utilizando herramientas que tradicionalmente han sido patrimonio de la investigación científica.

\section{FORMACIÓN DURA VERSUS FORMACIÓN BLANDA}

Otra tendencia a considerar para la tipificación de la demanda de profesionales universitarios por parte de las empresas, es el tipo de enfoque en la formación profesional, incluso dentro de un mismo campo. Es así como en casi todas las áreas y en particular en la Ingeniería existen carreras duras y carreras blandas, connotación que no necesariamente se relaciona con la calidad de la entidad formadora, 
ni con la del programa ofrecido, como tampoco con la capacidad de las personas que lo cursan y aprueban.

Se trata del enfoque que se utiliza. Un ejemplo son los programas de formación en Ingeniería Industrial, que enfatizan aspectos del ejercicio práctico, de formación en laboratorio y en plantas de producción; otros ponen el acento más bien en la periferia, potenciando aspectos más generales de planificación y aplicación de Teoría de Sistemas.

La distinción se relaciona con el real contacto que un profesional tiene o deja de tener con la realidad y la práctica durante su formación. Es por ello que se dice que existen ingenieros industriales de formación dura y blanda. Una Ingeniería blanda podría ser una que, en general, descansa en la formación por la vía de la simulación y, en menor grado, por la práctica concreta del oficio. Este fenómeno es más propio de la Ingeniería que de otras profesiones. Es inconcebible un enfoque blando en temáticas como la Medicina, aunque hay diversos grados de relación con el medio profesional en carreras como la Arquitectura.

En cuanto a la conveniencia y pertinencia de la formación para la incorporación al mundo empresarial, una formación dura entrega herramientas más concretas para abordar problemas también específicos. Por el contrario, una formación blanda aporta soluciones de aplicación más general, aunque menos relacionadas con la profesión específica. Es decir, si se trata de un profesional de la Ingeniería, esa persona tendrá un perfil general de ingeniero como producto de una formación más blanda y uno más especializado, si proviene de una realidad formativa más dura.

La decisión acerca de cuál perfil es más o menos apropiado para la realidad de una empresa determinada, descansa obviamente en el tipo de cargo que se procura llenar. Sin embargo, si se pretende tener una idea de la adecuación general de un profesional a los requerimientos de la empresa solo a partir de su formación de pregrado, las tendencias actuales señalan que, quizás, un generalista sea más adecuado, aunque si se trata de uno con formación en ambos niveles, pre y posgrado, la mezcla puede resultar en una combinación aun más apropiada. 


\section{¿PROFESIONES O SABERES?}

Lo analizado respecto de enfoques duros y blandos conduce a una consideración adicional, si acaso hoy las profesiones como tales son relevantes o si más bien se procura diferenciar la preparación de las personas sobre la base de saberes. La distinción percibe la diferencia entre un primer enfoque, identificado por la posesión de un conjunto relativamente rígido de conocimientos y destrezas que configuran un prototipo de profesional, el cual responde a un molde relativamente consensual.

El segundo corresponde más bien a saberes, conocimientos y destrezas, que tienen existencia independiente. Estos deberán ser considerados por el potencial empleador a la hora de seleccionar una persona para una determinada posición.

La tendencia a futuro apunta más bien a la segunda. Ella permitirá, por una parte, que las personas escojan los saberes más relevantes a sus inclinaciones y aptitudes y que, por otra, puedan complementar esa formación inicial, para evolucionar de acuerdo a las demandas del mercado del empleo.

Es así como las visiones que hace pocos años se consideraban propias de la literatura de anticipación, hoy sugieren que en un futuro próximo la educación formal ofrecerá un número muy grande de opciones, de modo que cada persona podrá estructurar su formación prácticamente sin limitaciones. Más aún, la tendencia que se va definiendo en muchos países desarrollados es que la educación informal empieza ya a tomar una importancia notable, en particular orientada a satisfacer las necesidades de los adultos. Es también previsible que las actuales posibilidades de certificación, hoy exclusivas de la educación formal, alcancen en corto plazo a los esquemas informales. Es posible concluir que la tendencia general favorece la educación continua, entendida como un proceso permanente y personalizado, el cual será capaz de responder con mayor flexibilidad a las demandas cada vez más cambiantes del mercado.

\section{COMENTARIOS ACERCA DE ALGUNOS RASGOS DESEABLES PARA LOS FUTUROS PROFESIONALES CHILENOS}

La globalización obligará al profesional chileno de los próximos años, 
y en particular a quien labore en la empresa privada, a adecuar su perfil al que hoy impera en la gran mayoría de los países, en particular en los más desarrollados.

Quizás la flexibilidad sea el rasgo más importante, es decir, la capacidad de adecuarse y, lo que es más, de responder a demandas cambiantes. Implica buscar y, eventualmente, diseñar vías de solución diferentes a problemas también nuevos, aunque sin transgredir los aspectos centrales de la doctrina de la empresa o actividad en que se inserte.

Otro aspecto primordial es la capacidad de emprender, la cual se liga estrechamente con tomar riesgos. Ésta, a su vez, es inseparable de la capacidad de análisis de fortalezas y debilidades, como también la de predicción de resultados, con muy poca información y en plazos cada vez más estrechos.

Dentro de los rasgos a considerar está una gran capacidad de adecuarse a formas de pensar y de ser diferentes. Requiere de un manejo fluido de lenguajes, el verbal, ojalá en varios idiomas, el matemático, además del informático, todos en el extremo formal; en el extremo más informal y complejo, la comprensión de realidades distintas, resultado del conocimiento y una amplia cultura general. Condición fundamental es mantener una disposición, unida a la motivación, por aprender de manera permanente, apoyada por un desarrollo de la metacognición, ello es de la autoconciencia de la propia capacidad individual y personalizada de aprender.

Resulta imposible agotar la lista de rasgos sin hacer mención a un aspecto cuya desmedrada situación es hoy evidente, no solo en Chile sino que en la región latinoamericana, como es la ausencia relativa de una cultura de la moral y ética en las actividades profesionales. En un momento en el cual la corrupción afecta a gobiernos y empresas, no es posible omitirla en el presente recuento. Por años fue considerada una materia propia del ámbito familiar y casi íntimo de la formación de la persona, pero el creciente desarrollo del conocimiento acerca de la interacción entre lo cognitivo y lo valórico lleva a exigir que de las instituciones educativas aborden el tema de manera sistemática al momento de estructurar la formación de los futuros profesionales. 
Considerando que esta no es solo una materia de opción religiosa, debe constituirse en un ingrediente fundamental de la formación profesional, se entregue ésta en un entorno confesional o laico.

Un aspecto final, que puede parecer casi un buen deseo, es evidente hoy en nuestro país. Es la resiliencia, la cual implica capacidad para enfrentar las dificultades, de rehacerse luego de una derrota. En fin, de abordar las propias actividades personales y profesionales con optimismo y espíritu de superación.

En la actualidad, es posible reconocer en nuestro país a quienes, gracias a una actitud como la mencionada, han sido capaces de sostener su actividad en tiempos de crisis y por ello ir en la avanzada al momento en que se vislumbra una recuperación, con la ventaja adicional de estar en mejor pie para aprovechar las nuevas condiciones.

\section{LA UNIVERSIDAD FRENTE A LA EVOLUCIÓN DE LA DEMANDA DE PROFESIONALES EN CHILE.}

Las instituciones universitarias nunca se han caracterizado por presentar una reacción rápida ni adecuada a la demanda de profesionales por parte del mercado laboral. Más aún, escudándose en una mal entendida concepción de la libertad académica se han negado a adoptar medidas de autorregulación, frente a la proliferación y permanencia de carreras orientadas al desempeño de profesiones sin mayor demanda en el mercado. Lo planteado no obsta para que se mantengan áreas de desarrollo académico en temas que no tienen, necesariamente, demanda en el mercado del empleo.

Sin duda, resulta difícilmente aceptable que se conserven abiertas como opciones profesionales áreas sin demanda laboral. Por ejemplo, no es comprensible que frente a la demanda decreciente de profesores en ciertos idiomas, hoy están ausentes en la enseñanza formal, sigan abiertas estas opciones, aunque es también difícil de comprender la desaparición de las áreas académicas de investigación y desarrollo en tales idiomas. Es importante no perder de vista que la universidades no constituyen solamente entes dedicados a la docencia, sino que la investigación debe ser un aspecto central de su actividad. 
El tema del financiamiento es un segundo elemento a considerar al momento de abordar la capacidad de reacción de las universidades frente a la demanda de profesionales. Cuando se exige a las casas de estudio no reajustar aranceles, otorgar financiamiento a todos los estudiantes y, además, no incurrir en déficit, es muy difícil esperar que bajo una situación de presión financiera sin salida razonable y razonada sean capaces de adecuarse a la demanda cambiante de profesionales por parte del mercado laboral.

La clave para permitir una adecuación de las universidades a las demandas del mercado de profesionales requiere, por una parte, estabilidad para ellas, realismo en todos sus miembros y pragmatismo al momento de enfrentar los cambios. 
REFERENCIA BiBLIOGRÁFICA

OECD, París, 2000. Organisation of Economic Co-operation and Development and Statistics Canada. Literacy in the Information Age. Final Report of the International Literacy Survey. 
